\title{
Utility of Easy Z-Score Imaging System- Assisted SPECT in Detecting Onset Age-Dependent Decreases in Cerebral Blood Flow in the Posterior Cingulate Cortex, Precuneus, and Parietal Lobe in Alzheimer's Disease with Amyloid Accumulation
}

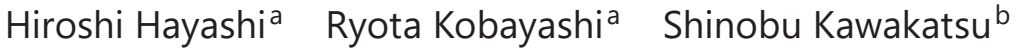 \\ Daichi Morioka $^{a} \quad$ Koichi Otani ${ }^{a}$ \\ ${ }^{a}$ Department of Psychiatry, Yamagata University School of Medicine, Yamagata, Japan; \\ ${ }^{b}$ Department of Neuropsychiatry, Aizu Medical Center, Fukushima Medical University, \\ Aizuwakamatsu, Japan
}

\section{Keywords}

Easy Z-score imaging system · Single-photon emission computed tomography · Alzheimer's disease $\cdot$ Onset age $\cdot{ }^{11} \mathrm{C}$-Pittsburgh compound B-positron emission tomography

\begin{abstract}
Background: Easy Z-score imaging system (eZIS)-assisted SPECT accurately detects decreases in cerebral blood flow in the posterior cingulate cortex (PCC), precuneus, and parietal lobe, the cerebral regions deeply implicated in Alzheimer's disease (AD). Several studies suggested onset age-dependent decreases in cerebral blood flow in these regions in AD, but these studies did not screen for amyloid accumulation, suggesting inclusion of non-AD patients in their subjects. Objective: By applying eZIS-SPECT to patients with amyloid deposition, it was the aim of this study to clarify onset age-dependent decreases in cerebral blood flow in the regions critical to AD. Methods: We retrospectively analyzed eZIS-SPECT data on 34 AD patients with amyloid retention confirmed by ${ }^{11} \mathrm{C}$-Pittsburgh compound B-PET. The subjects were divided into an early-onset group $(n=16)$ and a late-onset group $(n=18)$. The three indicators of the eZIS that had discriminated between AD patients and normal controls in previous studies were compared between the two groups. Results: The mean values for the respective indicators were significantly higher in the early-onset group than in the late-onset group. Also, the proportion of patients with abnormalities in all indicators was significantly higher in the
\end{abstract}


early-onset group (93.8\%) than in the late-onset group (50.0\%). Conclusions: The present study, applying eZIS-SPECT to amyloid-positive AD patients, suggests that reduced cerebral blood flow in the PCC, precuneus, and parietal lobe is more pronounced in the early-onset type than in the late-onset type of the disease.

(C) 2020 The Author(s)

Published by S. Karger AG, Basel

\section{Introduction}

Alzheimer's disease (AD) is the most common progressive neurodegenerative disease that causes dementia [1]. The posterior cingulate cortex (PCC), precuneus, and parietal lobe are considered to be deeply implicated in the pathophysiology of $\mathrm{AD}$, since these regions show reduced glucose metabolism [2] and regional cerebral blood flow (rCBF) [3] from an early stage of the disease. Meanwhile, the importance of amyloid deposition in the pathophysiology of $\mathrm{AD}$ is increasingly recognized, as reflected by the inclusion of this index in the diagnostic criteria for the disease [4]. ${ }^{11} \mathrm{C}$-Pittsburgh compound $\mathrm{B}(\mathrm{PiB})$ is a biomarker for amyloid accumulation, and the most reliable tracer for amyloid positron emission tomography (PET) [5]. Previous studies using amyloid PET [6, 7] showed that approximately $20-40 \%$ of patients with probable AD based on the clinical diagnostic criteria were amyloid negative, indicating the usefulness of this imaging method to discriminate between AD and non-AD.

Single-photon emission computed tomography (SPECT) is an imaging technique that is widely used in diagnosing dementia including $\mathrm{AD}[3,8]$. However, it has some difficulty in visually detecting a reduction of cerebral blood flow in the regions mentioned before, since they are inherently high in cerebral blood flow [9]. The easy Z-score imaging system (eZIS) (FUJIFILM RI Pharma, Co., Ltd., Tokyo, Japan) objectively evaluates the degree of reduction in rCBF in the PCC, precuneus, and parietal lobe, and automatically calculates three indicators that discriminate between AD patients and normal controls [10]. Because of these advantages, eZIS-SPECT is increasingly used for the diagnosis of AD [11,12].

Previous studies using SPECT suggested onset age-dependent decreases in $\mathrm{rCBF}$ in $\mathrm{AD}$ $[8,13]$. However, in none of these studies, amyloid PET was conducted, suggesting inclusion of substantial numbers of non-AD patients in their subjects. Therefore, the present study using eZIS-SPECT examined whether the degree of reduction in $\mathrm{rCBF}$ in the PCC, precuneus, and parietal lobe differs depending on the age at onset in AD with PiB positivity.

\section{Subjects and Methods}

\section{Subjects}

In this retrospective study, we firstly checked all patients with dementia who underwent PiB-PET at Yamagata University Hospital between September 2013 and March 2018. All of these patients had undergone magnetic resonance imaging (MRI) and eZIS-SPECT prior to PiB-PET. There were 68 patients with PiB retention and fulfilling the diagnostic criteria for probable AD dementia with amnestic presentation by the National Institute on Aging-Alzheimer's Association [4]. Next, we excluded patients who were taking antidementia drugs and who had a history of psychiatric disorders, severe physical illness, a Mini-Mental State Examination (MMSE) score < 15, and presence of cerebrovascular lesions and extensive white matter abnormalities. Thirty-four patients remained, and they constituted the subjects of this study.

The mean age $( \pm S D)$ was $69.4 \pm 9.0$ years, and the sex ratio (male:female) was balanced (15:19). The subjects were divided into an early-onset group with an onset age of $<65$ years $(n=16)$ and a late-onset group with an onset age of $\geq 65$ years $(n=18)$.

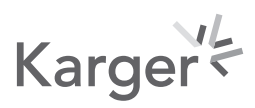


Dementia and Geriatric Cognitive Disorders Extra

Table 1. Demographic and clinical data for the early-onset and late-onset groups

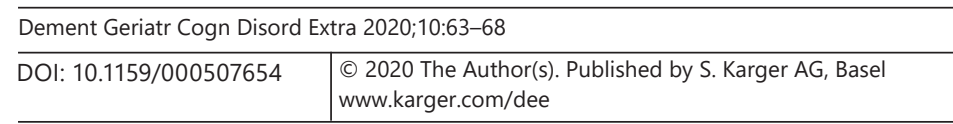

Hayashi et al.: Easy Z-Score Imaging System in Alzheimer's Disease

\begin{tabular}{lccc}
\hline & $\begin{array}{l}\text { Early-onset } \\
\text { group } \\
(n=16)\end{array}$ & $\begin{array}{l}\text { Late-onset } \\
\text { group } \\
(n=18)\end{array}$ & $p$ value \\
\hline Age, years & $61.2(5.1)$ & $76.7(4.1)$ & 0.000 \\
Sex (male/female) & $8 / 8$ & $7 / 11$ & 0.515 \\
Educational history, years & $12.1(1.8)$ & $12.4(1.6)$ & 0.654 \\
Disease duration, years & $3.3(1.6)$ & $3.3(2.6)$ & 0.974 \\
MMSE score (max. 30) & $23.1(3.3)$ & $21.3(4.4)$ & 0.204 \\
mcSUVR & $1.65(0.15)$ & $1.62(0.18)$ & 0.732 \\
\hline
\end{tabular}

Values are presented as mean (SD) or $n$. Differences between groups were assessed using Student's $t$ test (age, educational history, disease duration, and MMSE score) and Fisher's exact test (sex ratio). MMSE, Mini-Mental State Examination; mcSUVR, mean cortical standardized uptake value ratio.

eZIS-SPECT

Firstly, the patients received an intravenous injection of $600 \mathrm{MBq}$ of ${ }^{99 \mathrm{~m}} \mathrm{Tc}$-ethylcysteinate dimer (FUJIFILM Toyama Chemical, Co., Ltd., Tokyo, Japan). After 6 min 50 s, SPECT data were obtained in a $128 \times 128$ matrix on a Symbia T2 (Siemens Healthcare, Erlangen, Germany) mounted with low-energy, high-resolution collimators. Images were reconstructed by the filtered back-projection method with combined Chang attenuation and scatter correction. Statistical imaging analysis was performed with the eZIS software (FUJIFILM Toyama Chemical). $Z$-scores were calculated using the statistical parametric mapping 8 and the eZIS program. After anatomical standardization, a $Z$-score map for each SPECT image was drawn while referring to the mean and SD of age-matched normal controls already built into the eZIS program. After voxel normalization to global means or cerebellar values, a voxel-by-voxel $Z$-score was calculated and evaluated as follows: $Z$-score $=([$ control mean $]-$ [individual value])/(control SD), and a $Z$-score of $>2$ SDs was considered to indicate a reduction in $\mathrm{rCBF}$.

A specific volume of interest (VOI) was set on the PCC, precuneus, and parietal lobe in the eZIS program. The three indicators that had discriminated between AD patients and normal controls in previous studies [10-12] were automatically calculated. The severity of the decrease in $\mathrm{rCBF}$ was obtained from the averaged $Z$-score in the VOI. The extent of significantly decreased rCBF was obtained from the rate of voxels with a $Z$-score $>2$ in the VOI. The ratio of the extent of significantly decreased $\mathrm{rCBF}$ in the VOI to that in the whole brain was also calculated. This ratio indicates the specificity of $\mathrm{rCBF}$ reduction in a VOI compared with that in the whole brain. Cutoff values for likely AD were $>1.19$ for the severity, $>14.2 \%$ for the extent, and $>2.22$ for the ratio [10]. Previous studies demonstrated that patients with abnormalities in all indicators exhibited PiB retention by PiB-PET $[11,12]$.

PiB-PET

PiB-PET scans were performed using a PET/CT scanner (Biograph mCT; Siemens Healthineers, Tokyo, Japan) in 3D scanning mode. PiB was injected intravenously at a dose of $555 \pm$ $185 \mathrm{MBq}$, immediately followed by a 70-min dynamic acquisition. PET images acquired 50-70 min after injection (300 s $\times 4$ frames) were used. The images were analyzed with the PMOD software (version 3.409; PMOD Technologies Ltd., Zurich, Switzerland). The mean cortical standardized uptake value ( $\mathrm{mcSUV}$ ) was calculated for all brain regions examined. The mcSUV ratio (mcSUVR) was generated by normalizing the mcSUV to the cerebellar cortex SUV. Subjects with an mcSUVR $>1.4$ were classified as $\mathrm{PiB}$ positive [14]. 
Table 2. Three indicators of the eZIS in the early-onset and lateonset groups

Hayashi et al.: Easy Z-Score Imaging System in Alzheimer's Disease

\begin{tabular}{lcll}
\hline & $\begin{array}{l}\text { Early-onset } \\
\text { group } \\
(n=16)\end{array}$ & $\begin{array}{l}\text { Late-onset } \\
\text { group } \\
(n=18)\end{array}$ & $p$ value \\
\hline $\begin{array}{lccc}\text { Severity } \\
\text { Extent }\end{array}$ & $2.50(0.84)$ & $1.42(0.49)$ & 0.000 \\
$\begin{array}{l}\text { Ratio } \\
\text { Abnormalities in all } \\
\text { indicators }\end{array}$ & $52.3(22.3)$ & $20.4(15.6)$ & 0.000 \\
& $4.05(1.57)$ & $2.68(1.96)$ & 0.033 \\
\hline
\end{tabular}

Values are presented as mean (SD) or $n(\%)$. Differences between groups were assessed using Student's $t$ test (severity, extent, and ratio) and Fisher's exact test (rate of patients with abnormalities in all indicators). eZIS, easy Z-score imaging system.

\section{Statistical Analysis}

Student's $t$ test was used to compare demographic data except the sex ratio, clinical data, and three indicators of the eZIS of the subjects between the early-onset and the late-onset group. Fisher's exact test was used to compare the sex ratio and the rate of patients with abnormalities in all indicators of the eZIS between the two groups. Statistical analyses were performed using the SPSS software (version 25), and a $p$ value $<0.05$ was considered statistically significant.

\section{Results}

Table 1 shows the demographic and clinical data for the early-onset and late-onset groups. No significant differences were found regarding sex ratio, educational history, disease duration, MMSE score, or mcSUVR value between the two groups.

Table 2 shows the three indicators of the eZIS in the early-onset and late-onset groups. The mean values of severity, extent, and ratio were significantly higher in the early-onset group than in the late-onset group ( $p=0.000, p=0.000$, and $p=0.033$, respectively). The proportion of patients with abnormalities in all indicators was significantly $(p=0.008)$ higher in the early-onset group (93.8\%) than in the late-onset group (50.0\%).

\section{Discussion}

In contrast to previous SPECT studies on $\mathrm{AD}[8,13]$, the present study included confirmation of amyloid accumulation using PiB-PET in its protocol. Therefore, it is unlikely that our patients included those with non-AD such as argyrophilic grain dementia and senile dementia of the neurofibrillary tangle type, which exhibit similar clinical symptoms and hippocampal atrophy to $\operatorname{AD}[15,16]$.

By applying eZIS-SPECT to these amyloid-positive AD patients, the present study clarified onset age-dependent decreases in rCBF in the PCC, precuneus, and parietal lobe; the degree of reduction in these cerebral regions was significantly higher in the early-onset group than in the late-onset group. Recently, several neuroimaging studies have compared early-onset and lateonset $\mathrm{AD}$ with positivity for AD biomarkers. MRI studies have demonstrated that cerebral atrophy of the early-onset type was predominant in temporoparietal regions, especially the PCC, whereas that of the late-onset type was predominant in medial temporal regions $[17,18]$. 
In studies using fluorodeoxyglucose PET, the early-onset type showed hypometabolism in the precuneus and parietal lobe, more than the late-onset type did $[18,19]$. Furthermore, a neuropathological study revealed that the neurofibrillary tangle densities in the association cortices including the parietal cortex were higher in the early-onset type than in the late-onset type [20]. These studies suggest that pathophysiological abnormalities of the PCC, precuneus, and parietal lobes are more pronounced in the early-onset type than in the late-onset type. Therefore, our results complement these morphological, metabolic, and neuropathological findings.

From a clinical perspective, the present study suggests that eZIS-SPECT has high diagnostic utility for early-onset AD. On the other hand, it is recommended that this imaging method be applied to the diagnosis of late-onset $\mathrm{AD}$ with caution, probably in combination with morphological imaging such as MRI [21].

There are several limitations to this study. First, the sample size was small. Second, this study was performed retrospectively at one medical institution only, and the possibility of an unintended bias in patient selection cannot be ruled out entirely. Therefore, our results should be replicated in prospective studies with a larger number of subjects recruited from multiple facilities. Third, neuropathological confirmation was not performed.

In conclusion, the present study applying eZIS-SPECT to amyloid-positive AD suggests that the reduction in cerebral blood flow in the PCC, precuneus, and parietal lobe is more pronounced in the early-onset type than in the late-onset type of the disease.

\section{Acknowledgements}

We would like to thank all participants who took part in this study.

\section{Statement of Ethics}

The present study was approved by the Ethics Committees of Yamagata University School of Medicine and complied with the rules for human experimentation stated in the World Medical Association Declaration of Helsinki. All patients or their families provided written informed consent for participation.

\section{Disclosure Statement}

The authors have no conflicts of interest to declare.

\section{Funding Sources}

There were no funding sources.

\section{Author Contributions}

H.H. conceptualized the study, performed the neuropsychological assessment, analyzed the patient data, and drafted the manuscript; R.K., S.K., and D.M. performed the neuropsychological assessment and revised the manuscript; K.O. encouraged the study and revised the manuscript. All authors read and approved the final version of this manuscript.

\section{Karger'}




\section{References}

1 Cummings JL. Alzheimer's disease. N Engl J Med. 2004 Jul;351(1):56-67.

2 Del Sole A, Clerici F, Chiti A, Lecchi M, Mariani C, Maggiore L, et al. Individual cerebral metabolic deficits in Alzheimer's disease and amnestic mild cognitive impairment: an FDG PET study. Eur J Nucl Med Mol Imaging. 2008 Jul;35(7):1357-66.

3 Borroni B, Anchisi D, Paghera B, Vicini B, Kerrouche N, Garibotto V, et al. Combined 99mTc-ECD SPECT and neuropsychological studies in MCI for the assessment of conversion to AD. Neurobiol Aging. 2006 Jan;27(1): 24-31.

4 McKhann GM, Knopman DS, Chertkow H, Hyman BT, Jack CR Jr, Kawas CH, et al. The diagnosis of dementia due to Alzheimer's disease: recommendations from the National Institute on Aging-Alzheimer's Association workgroups on diagnostic guidelines for Alzheimer's disease. Alzheimers Dement. 2011 May;7(3):263-9.

5 Klunk WE, Engler H, Nordberg A, Wang Y, Blomqvist G, Holt DP, et al. Imaging brain amyloid in Alzheimer's disease with Pittsburgh Compound-B. Ann Neurol. 2004 Mar;55(3):306-19.

6 Ossenkoppele R, Prins ND, Pijnenburg YA, Lemstra AW, van der Flier WM, Adriaanse SF, et al. Impact of molecular imaging on the diagnostic process in a memory clinic. Alzheimers Dement. 2013 Jul; 9(4):414-21.

7 de Wilde A, van der Flier WM, Pelkmans W, Bouwman F, Verwer J, Groot C, et al. Association of amyloid positron emission tomography with changes in diagnosis and patient treatment in an unselected memory clinic cohort: the ABIDE Project. JAMA Neurol. 2018 Sep;75(9):1062-70.

8 Hanyu H, Shimuzu T, Tanaka Y, Takasaki M, Koizumi K, Abe K. Effect of age on regional cerebral blood flow patterns in Alzheimer's disease patients. J Neurol Sci. 2003 May;209(1-2):25-30.

9 Imabayashi E, Matsuda H, Asada T, Ohnishi T, Sakamoto S, Nakano S, et al. Superiority of 3-dimensional stereotactic surface projection analysis over visual inspection in discrimination of patients with very early Alzheimer's disease from controls using brain perfusion SPECT. J Nucl Med. 2004 Sep;45(9):1450-7.

10 Matsuda H, Mizumura S, Nagao T, Ota T, Iizuka T, Nemoto K, et al. Automated discrimination between very early Alzheimer disease and controls using an easy Z-score imaging system for multicenter brain perfusion single-photon emission tomography. AJNR Am J Neuroradiol. 2007 Apr;28(4):731-6.

11 Yokoyama S, Kajiya Y, Yoshinaga T, Tani A, Hirano H. Imaging discrepancies between magnetic resonance imaging and brain perfusion single-photon emission computed tomography in the diagnosis of Alzheimer's disease, and verification with amyloid positron emission tomography. Psychogeriatrics. 2014 Jun; 14(2): $110-7$.

12 Takemaru M, Kimura N, Abe Y, Goto M, Matsubara E. The evaluation of brain perfusion SPECT using an easy Z-score imaging system in the mild cognitive impairment subjects with brain amyloid- $\beta$ deposition. Clin Neurol Neurosurg. 2017 Sep;160:111-5.

13 Kemp PM, Holmes C, Hoffmann SM, Bolt L, Holmes R, Rowden J, et al. Alzheimer's disease: differences in technetium-99m HMPAO SPECT scan findings between early onset and late onset dementia. J Neurol Neurosurg Psychiatry. 2003 Jun; 74(6):715-9.

14 Murray ME, Lowe VJ, Graff-Radford NR, Liesinger AM, Cannon A, Przybelski SA, et al. Clinicopathologic and 11C-Pittsburgh compound B implications of Thal amyloid phase across the Alzheimer's disease spectrum. Brain. 2015 May;138(Pt 5):1370-81.

15 Ferrer I, Santpere G, van Leeuwen FW. Argyrophilic grain disease. Brain. 2008 Jun;131(Pt 6):1416-32.

16 Crary JF, Trojanowski JQ, Schneider JA, Abisambra JF, Abner EL, Alafuzoff I, et al. Primary age-related tauopathy (PART): a common pathology associated with human aging. Acta Neuropathol. 2014 Dec;128(6):755-66.

17 Dickerson BC, Brickhouse M, McGinnis S, Wolk DA. Alzheimer's disease: the influence of age on clinical heterogeneity through the human brain connectome. Alzheimers Dement (Amst). 2016 Dec;6(1):122-35.

18 Aziz AL, Giusiano B, Joubert S, Duprat L, Didic M, Gueriot C, et al. Difference in imaging biomarkers of neurodegeneration between early and late-onset amnestic Alzheimer's disease. Neurobiol Aging. 2017 Jun;54: 22-30.

19 Ossenkoppele R, Zwan MD, Tolboom N, van Assema DM, Adriaanse SF, Kloet RW, et al. Amyloid burden and metabolic function in early-onset Alzheimer's disease: parietal lobe involvement. Brain. 2012 Jul;135(Pt 7): 2115-25.

20 Murray ME, Graff-Radford NR, Ross OA, Petersen RC, Duara R, Dickson DW. Neuropathologically defined subtypes of Alzheimer's disease with distinct clinical characteristics: a retrospective study. Lancet Neurol. 2011 Sep;10(9):785-96.

21 Shibuya Y, Kawakatsu S, Hayashi H, Kobayashi R, Suzuki A, Sato C, et al. Comparison of entorhinal cortex atrophy between early-onset and late-onset Alzheimer's disease using the VSRAD, a specific and sensitive voxel-based morphometry. Int J Geriatr Psychiatry. 2013 Apr;28(4):372-6. 